Chiffres réels

(1)

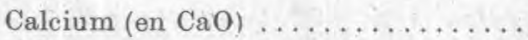

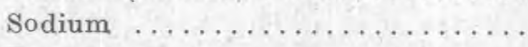

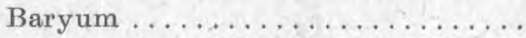

Aluminium (en $\mathrm{Al}^{2} \mathrm{O}^{3}$ )

$2^{\circ}$ Exprimés en substances reconstituées

D'après les

chiffres réels

Caséine industrielle $\ldots \ldots \ldots \ldots \ldots$.

Gélatine en poudre .............

Chaux éteinte pure .............

Fluorure de sodium .............

Phosphate trisodique $\left(\mathrm{a} 12 \mathrm{H}^{2} \mathrm{O}\right) \ldots$.

Carbonate de sodium anhydre ......

Borate de sodium (à $\left.10 \mathrm{H}^{2} \mathrm{O}\right) \ldots \ldots \ldots$

Silicate de sodium sec............

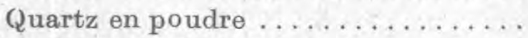

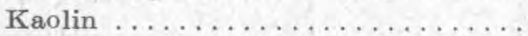

Sulfate de baryum .............
Chiffres trouvés à l'analyse du mélange

$$
\begin{array}{r}
5,46 \\
10,37 \\
2,75 \\
2,02
\end{array}
$$

par le calcul.

D'après les chiffres trouvés à l'analyse

\begin{tabular}{rr}
25,0 & 24,83 \\
25,0 & 25,62 \\
7,20 & 7,21 \\
7,43 & 7,15 \\
5,30 & 5,12 \\
4,77 & 4,87 \\
5,23 & 5,29 \\
7,39 & 7,17 \\
2,47 & 2,48 \\
5,14 & 5,18 \\
4,74 & 4,77 \\
\hline- & - \\
99,67 & 99,69
\end{tabular}

Comme on le voit, les méthodes d'analyse proposées donnent d'assez bons résultats, même dans le cas d'un mélange aussi complexe que celui-ci. Dans les cas courants (mélanges de 3 ou 4 substances au plus) l'analyse est beaucoup plus facile et les résultats obtenus sont encore meilleurs.

\title{
ANTAGONISIME DU COLIBACILLE ET DES BACTÉRIES PUTRIDES DANS LE LAIT CONTAMINÉ (2)
}

\author{
par
}

\section{A. ROCHAIX et F. SIMON}

On sait que parmi les microbes ayant une signification de contamination fécale, que l'on a l'habitude de rechercher dans les liquides comme l'eau, le lait, etc., se trouve au premier plan le colibacille, auquel on a adjoint depuis quelques années les "bactéries putrides". Ces dernières sont des microbes paraissant

(1) Voir note précédente, page 143.

(2) Ass. Microb. de langue française, 2-12-1943; Ann. Inst. Pasteur, 1944, LXX, 313. 
appartenir auxespèces les plus variées mais présentant des réactions biologiques communes les rendant suspects,en particulier dégageant, aux dépens des matières albuminoïdes du milieu, de l'hydrogène sulfuré et du sulfhydrate d'ammoniaque. Ces éléments, qui donnent des colonies noires sur la gélose au sous-acétate de plomb, constituent aussi un élément très significatif de pollution et on ne manque pas de les rechercher.

Or, il existe entre colibacille et "bactéries putrides » pouvant exister dans l'eau un antagonisme sur lequel l'un de nous a déjà attiré l'attention (1). Au point de vue du contrôle de la qualité bactériologique de ce liquide, ce fait présente une grande importance. Si l'on ne recherche pas les deux catégories de microbes en même temps, si l'on se contente de rechercher le colibacille seul, comme indice de contamination, il peut être absent ou ne se trouver qu'en quantité insignifiante, alors qu'une contamination très élevée peut être révélée par les résultats positifs de la recherche des "bactéries putrides". Mais, dans l'eau, le phénomène ne se présente pas très fréquemment. Nous ne l'avons noté que quinze fois sur 1.200 examens. Au cours d'examens de contrôle de pureté bactériologique d'échantillons de lait, nous avons observé cet antagonisme avec une fréquence et une intensité infiniment plus grandes. Sur 157 analyses d'échantillons de lait, reconnus contaminés, dans 37 cas le colibacille n'existait pas, alors que les colonies de "bactéries putrides » se trouvaient en grand nombre. Voici quelques exemples:

\begin{tabular}{|c|c|}
\hline Colibacille & $\begin{array}{c}\text { Bactéries putrides } \\
\text { Au litre }\end{array}$ \\
\hline $0 \ldots$ & 100.000 \\
\hline 0 & 50.000 \\
\hline $0 \ldots$ & 250.000 \\
\hline $0 \ldots$ & 200.000 \\
\hline 0 & 250.000 \\
\hline $0 \ldots$ & 50.000 \\
\hline $0 \ldots$ & 150.000 \\
\hline 0 & 50.000 \\
\hline $0 \ldots$ & 250.000 \\
\hline $0 \ldots$ & 250.000 \\
\hline
\end{tabular}

D'autre part, fait que nous n'avions pas observé dans l'eau, où c'était toujours le colibacille qui disparaissait devant les bactéries putrides, nous avons observé la disparition des bactéries putrides devant le colibacille dans 15 cas. Voici quelques exemples :

(1) A. Rochaix et Mile Urtinetre. Comptes rendus Soc. de Biol., 1931, LXVI, 669. 
Colibacille Bactéries putrides

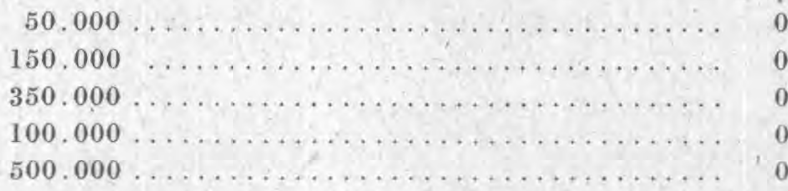

0
0
0
0

Dans une troisième catégorie de cas, sans que l'un ou l'autre des deux microbes ait complètement disparu, l'un domine l'autre a vec un écart considérable :

\begin{tabular}{|c|c|c|c|}
\hline Colibacille & $\begin{array}{l}\text { Bactéries } \\
\text { putrides }\end{array}$ & Colibacille & $\begin{array}{l}\text { Bactéries } \\
\text { putrides }\end{array}$ \\
\hline 50.000 . & 400.000 & 500.000. & 150.000 \\
\hline 200.000 . & 450,000 & 500.000. & 100.000 \\
\hline 50.000 & 350.000 & .450 .000 & 200.000 \\
\hline 50.000. & $\tilde{500.000}$ & 500.000 . & 200.000 \\
\hline 100.000 & 500.000 & 500.000. & 150.000 \\
\hline 150.000 & 500.000 & 500.000 . & 50.000 \\
\hline & etc.... & & etc... \\
\hline
\end{tabular}

Cette dernière catégorie de faits est moins démonstrative, mais étant donné la différence du nombre des colonies (nous n'avons retenu que les écarts d'au moins 200.000), il semble qu'on puisse en tenir compte. Dans 19 cas, le nombre des bactéries putrides dépassait de plus de 200,000 celui des colibacilles; dans 12 cas, c'étaient ces derniers microbes qui étaient les plus nombreux.

La proportion, si l'on ne tient pas compte de cette dernière catégorie, est donc de $33,12 \%$. Si l'on tient compte de la troisième catégorie, la proportion monte à $52,96 \%$, alors que, dans l'eau, la proportion n'atteint que $1,24 \%$. La recherehe des bactéries putrides apparaît done pour le lait d'une importance de premier ordre. L'hydrolyse de la caséine du lait pour 100 a permis d'isoler un certain nombre d'acides aminés contenant du soufre, eystine, eystéine et leurs homologues supérieurs (homocystine, homocystéine), méthionine. Il n'est pas étonnant que les bactéries, ensemencées avec les dilutions de lait sur les milieux de recherche, dégagent plus facilement que lorsqu'il s'agit de l'eau, de l'hydrogène sulfuré. C'est un phénomène analogue à celui qui se passe dans la recherche de l'hydrogène sulfuré avec des microbes isolés en cultures pures, quand on ajoute au milieu de culture du soufre pulvérulent; cette addition augmente la production de l'hydrogène sulfuré.

Quant au mécanisme de l'antagonisme, il est difficile à préciser. On pourrait penser, la production de l'hydrogène sulfuré étant intense, qu'il exerce une action bactéricide particulièrement 
efficace sur le colibacille. Mais cette explication ne peut être retenue: Outre que l'on doit admettre que le colibacille a dû s'accoutumer à vivre en commensal avee les bactéries putrides dans l'intestin de l'homme et des animaux, les expériences de Douris et BECK ne permettent pas de l'accepter. Ces Auteurs (1), en effet, ont montrê que sur toute une série de microbes, soumis en cultures saturées, ì plusieurs reprises, d' $H^{2} \mathrm{~S}$, à l'action bactéricide de ce gaz, e'est le colibacille qui résiste le plus longtemps. D'ailleurs nous avons vu que dans un certain nombre de cas, moins nombreux il est vrai (15 contre 37), ce sont les bactéries putrides qui disparaissent devant le colibacille. Dans ce dernier cas, est-ce l'acidité développée par le colibacille qui intervient? Des recherches sont en cours pour pénétrer le mécanisme de cet antagonisme, vraisemblablement complexe.

(Institut Bactériologique de Lyon.)

\section{BIBLIOGRAPHIE ANALYTIQUE}

\section{LES LIVRES}

\section{Mulder (H.). - De Bepaling van Phosphatiden in Karnemelk} (Estimation des phospholipides du babeurre). Rijkslandbouwproefstation te Hoorn. Verslagen van Landbouwkundige onderzoekingen, t. XII C, $\mathrm{n}^{\circ} 48,1942$.

Après , une revue bibliographique, l'A. expose les résultats obtenus d'après trois méthodes différentes auxquelles il accorde une valeur sensible. ment égale.

La méthode de Brodrick-Pittard apparait très pratique.

Lá méthode d'Holwerda convient pour le babeurre acide ; elle convient moins pour le babeurre doux et pour d'autres produits laitiers dans lesquels apparaissent des composants dérivés capables de provoquer des erreurs d'appréciation. Contrairement aux conelusions posées par HoLWERDA, M. trouve des phospholipides dans le lait écrémé $(0,01$ à $0,015 \%)$. En eonséquence, la correction concernant les composants dérivés non lipidiques, correction nécessaire avec l'emploi de cette méthode, ne peut pas être établie par l'estimation des phospholipides dans le lait écrémé, mais doit être déterminée sur un ultra-filtrat du produit laitier considéré.

Dans la troisième méthode, I'A. utilisa la technique de Röse-Gottlieb pour l'extraction de la matière grasse, tandis que le taux en phosphore de l'extrait était apprécié après traitement du résidu selon Neumann aveo le réactif de Medinger d'après la méthode pratiquée par Holwerda. Il fut estimé que les phospholipides du lait contenaient $4 \%$ de phosphore. Cette

(1) Douris et Beok. Ann. Inst. Pasteur, 1931, XLVI, 73. 